\title{
La classe des adjectifs dans un créole français : le cas du mauricien
}

\author{
Alleesaib, Muhsina \\ UMR 7023 Structures Formelles du Langage \& Université Charles de Gaulle Lille 3 \\ \{muhsina.alleesaib \}@univ-lille3.fr
}

\section{Introduction}

La question à laquelle tente de répondre cet article est l'existence de propriétés distributionnelles qui permettent de distinguer une classe adjectivale en mauricien. Dans plusieurs créoles, les lexèmes qui sont historiquement dérivés d'adjectifs se combinent directement avec un marqueur d'aspect progressif (cf. Alleyne 1996: 64 pour les créoles français). Ce serait une preuve que les adjectifs forment une sousclasse d'une catégorie plus vaste qu'on pourrait appeler prédicat afin d'éviter le terme verbe. Holm (2000) n'hésite pas à employer le terme adjectival verbs. Les concepteurs du Atlas of Pidgin and Creole Structures ne tranchent pas sur la question et emploient le terme adjectif.

Cette question intéresse les spécialistes des créoles parce que la non-existence d'une classe adjectivale serait une preuve supplémentaire que les langues créoles sont issues d'une variété simplifiée, appelée pidgin ou, parfois, basic variety. La perte de la morphologie aurait pour résultat une réduction dans le nombre de classes distinctes. Le débat sur la classe adjectivale pourrait aussi intéresser les linguistes qui soutiennent que les langues créoles constituent une classe typologique. L'absence d'une classe adjectivale pourrait constituer un trait structurel partagé par les langues créoles.

Certains linguistes remettent en question l'existence de catégories universelles et proposent des catégories qui sont propres aux langues elles-mêmes ou aux familles de langue (cf. Haspelmath 2007). D'autres linguistes admettent que la classe des adjectifs existe dans des langues très éloignées typologiquement des langues européennes, mais que celle-ci ne compte pas plus d'une dizaine de mots. C'est la position de Dixon (2004) et de Véronique (2000) pour les créoles français. Cette conception est à l'opposé de celle de Baker (2003) qui tente de prouver que chaque langue possède une classe adjectivale malgré les différences entre les langues.

Dans cet article, je mets en évidence le fait que les critères qui permettent de distinguer les adjectifs en anglais ne sont pas applicables au mauricien. La comparaison avec l'anglais est pertinente parce que les adjectifs en anglais ne s'accordent pas avec le nom. Je présente quelques faits sur la distribution des verbes-copules et de certains adverbes qui montrent qu'il y a lieu de distinguer la classe des adjectifs de celle des verbes. Je soutiens par ailleurs que la possibilité de se construire directement avec le progressif ne s'applique qu'à un petit nombre d'adjectifs. La compatibilité avec le progressif ne saurait être un argument pour classer les adjectifs dans la même catégorie que les verbes. 
L'article est organisé comme suit : dans la section 2, je résume les propriétés caractéristiques des adjectifs tels qu'ils sont présentés pour l'anglais. La section 3 est consacrée aux points communs entre les verbes et les adjectifs en mauricien. Je présente les données qui distinguent les adjectifs des verbes en mauricien dans la section 4 .

\section{Les propriétés distinctives des adjectifs}

Dans cette section, je présente quelques propriétés des adjectifs dans les langues les plus étudiées: l'apparition après une copule, la combinaison avec les verbes épistémiques, les constructions résultatives, les expressions de degré et la modification directe. Il ne s'agira pas ici de critères sémantiques (voir Dixon 1977 et Dixon $2004: 4$ sur les champs sémantiques propres aux adjectifs.)

Dans certaines langues, les adjectifs doivent apparaitre après une copule lorsqu'ils sont en position de prédicats (être en français et be en anglais). Etant donné que le mauricien n'emploie pas de copule pour les constructions prédicatives, on ne peut pas en faire critère distinctif (cf. section 3 pour un exemple).

En anglais, contrairement aux noms et aux verbes, les adjectifs peuvent être le complément de verbes épistémiques seem et appear 'paraître, apparaître', comme dans 'The price seems reasonable.' Comme sembler en français nécessité le verbe être (Ce prix semble être raisonnable), ce critère semble être propre à l'anglais. Je laisserai de côté ce critère pour des recherches ultérieures.

Dans beacoup de langues, les adjectifs peuvent modifier le nom directement (Baker 2003: 252-256; Dixon 2004: 19-20). Selon Baker, pour qu'un nom ou un verbe puisse modifier un nom, il faut qu'il soit introduit par une projection fonctionnelle telle une préposition ou un pronom relatif. Dans le cadre théorique de Baker (le modèle des Principes et Paramètres), une projection fonctionelle peut être occupée par un mot fonctionnel tel une préposition, par un morphème de cas (le 's du génitif en anglais) ou par un morphème zéro.Baker reconnait cependant que le critère de la modification directe n'est pas universel. Une langue comme La langue Esclave (langue athapascane) exclue cette possibilité (Rice 1989 cité par Baker 2003:194).

La généralisation de Baker est valable dans la mesure où il existe des différences claires entre la modification adjectivale et les noms composés. Cette distinction n'apparait pas aussi clairement dans une langue comme le mauricien. Selon Baker (2003), les propriétés de l'accent tonique permettent de distinguer en anglais les noms composés de la modification adjectivale. A ce stade, je n'ai pas encore relevé de différences accentuelles entre la composition et la modification en mauricien.

Pour Kennedy (2012), la différence tient à la récursion. En anglais, les adjectifs peuvent se combiner de manière récursive avec des noms afin de former des constituants plus complexes. Les noms ont aussi la possibilité de modifier directement un autre nom. Cependant, ces structures sont analysées comme des constructions morphologiques, celle des noms composés (ou compound nouns).

La composition nom-nom se distingue de la modification adjectivale de la manière suivante en anglais: le sens du nom composé est imprévisible et dépend du contexte tandis que la modification adjectivale produit toujours une interprétation prédictible et qui est le produit de la combinaison du sens de l'adjectif et de celui du nom. La composition des noms en anglais serait un processus morphologique qui peut aboutir à la lexicalisation tandis que les constructions Adjectif + Nom seraient des constructions syntaxiques. Si on considère le français, il est vrai que les noms-composés café-théâtre, bande-son et 
bateau-vapeur aient une interprétation idiosyncrasique, ce qui est conforme à la généralisation de Kennedy. Il serait cependant erroné de considérer que toutes les constructions nom + adjectif en français sont des constructions syntaxiques. Des exemples tels que ressources humaines et mouvement social montrent que les combinaisons Nom+ Adjectif peuvent être lexicalisées.

La seconde différence entre la modification adjectivale et les noms-composés tient à l'ordre des mots. Lorsque plusieurs adjectifs modifient un seul nom, ces adjectifs peuvent être ordonnés de manière libre entre eux ( $a$ big old house, an old big house). Il n'en est pas de même avec les noms composés, puisqu'un adjectif ne peut pas intervenir entre le nom modifié et le nom modifieur. Les noms café et théâtre ne peuvent être séparés par un adjectif, comme le montre l'agrammaticalité de *un café immense théâtre.

Cette généralisation doit cependant être relativisée parce qu'elle ne s'applique pas aux adjectifs relationnels. L'adjectif social ne peut pas être séparé de mouvement tel qu'illustré en (6).

(1) Un mouvement social désorganisé - *un mouvement désorganisé social.

Le problème est résolu si on analyse mouvement social mais comme une séquence lexicalisée qui serait synonyme de grève plutôt que comme une relation de modification. Dans le cadre du présent travail, je vais limiter le champ de mon investigation aux adjectifs intersectifs.

Un critère souvent invoqué pour distinguer les adjectifs anglais est que certaines expressions, notamment so 'si', too 'trop', as 'aussi', very 'très', enough 'assez' ne se combinent qu'avec des adjectifs. Kennedy (2012) remarque aussi qu'en anglais, les adjectifs se construisent directement avec les expressions de degré (direct composition). Les exemples suivants démontrent que l'adjectif dependent se combine directement avec l'adverbe de degré too mais que cela est exclu pour le nom dependence ou le verbe depends on qui exigent l'insertion de much (Kennedy 2012, exemple 6).

(2) a. The country is too dependent on foreign oil. 'Le pays est trop dépendant sur le pétrole étranger.'

b. $\quad *$ The country too depends on foreign oil.

Cependant, une étude détaillée des expressions de degré par Doetjes (2008) démontre que des expressions de degré ayant les mêmes contraintes ne se retrouvent ni en français ni en néerlandais (cf. section 4). Notons aussi que la modification par des expressions de degré est une caractéristique propre aux adjectifs scalaires, et ne s'applique pas aux adjectifs non-scalaires. Les adjectifs relationnels tels social, carcéral ne sont pas concernés par ce critère.

Le fait qu'en anglais, la prédication seconde à interprétation résultative se construise avec les adjectifs sans l'emploi d'une préposition est une différence supplémentaire entre ceux-ci et les noms et les verbes (Baker 2003: 219-230). Les exemples en (33) montrent cette différence.

(3) a. They beat the metal flat.

'Ils ont battu le métal jusqu'à ce qu'il soit plat.' Litt. 'Ils ont battu le métal plat.'

b. *They beat the metal a sword.

c. $\quad *$ They polished the coin shine.

La structure conceptuelle de ce type de construction est selon Baker CAUSE X BE + Y, où Y est exprimé par l'adjectif et X un syntagme nominal (FAIRE en sorte que X soit Y). On ne dispose pas pour l'instant 
d'études détaillées sur l'interprétation résultative de la prédication seconde en mauricien qui permettent de vérifier cette généralisation.

Dans la section suivante, je présente les raisons pour lesquelles on a été amené à réduire les adjectifs prédicatifs et les verbes à une seule catégorie.

\section{Les adjectifs du mauricien en position prédicative}

La prédication averbale est construite comme la prédication verbale, c'est-à-dire sans élément remplissant la fonction de copule.

(4) Mala for dan latin. / Mala enn dokter.

M. fort PREP latin $M$. un médecin

'Mala est forte en latin. / Mala est un médecin.'

Les lexèmes dérivés d'adjectifs français occupent la même positon prédicative que les verbes. Il est aussi remarquable qu'ils se combinent directement avec la marque du passé $t i$.

(5) a. Mala ti for dan latin.

M. PAST fort PREP latin

b. Mala ti dormi anba.

M. PAST dormi par.terre

'Mala dormait par terre.'

Le mauricien permet aussi l'insertion de marqueurs d'aspect devant plusieurs adjectifs. Les deux marqueurs d'aspect du mauricien sont le perfectif (f)inn et le progressif pe. La combinaison d'un adjectif avec le perfectif est un processus productif.

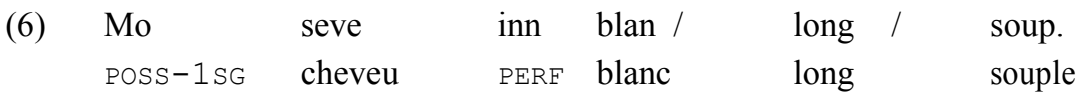

'Mes cheveux ont blanchi/ Mes cheveux sont plus longs. / Mes cheveux sont à présent souples.'

Certains auteurs considèrent que le fait que le marqueur progressif puisse se combiner avec des adjectifs est une preuve que ces derniers sont une sous-classe des verbes (cf. Alleyne 1996: 64 et Véronique 1983: 208). Cependant, Véronique (2000) remet en question cette généralisation. Cette question est traitée dans la section 4 .

Il n'existe pas de critères morphologiques qui permettent d'identifier une classe adjectivale. Le mauricien est presque dépourvu de morphologie dérivationnelle. Il n'existe pas de morphologie productive qui dérive des adjectifs des noms comme cela existe en français. Les lexèmes suivants sont des emprunts du français, et les règles de dérivation en - $a l$, -eux, ne sont pas productives.

(7) a. komersial (n'est pas dérivé de komers)

b. amoure ( n'est pas dérivé de lamour) [mauricien]

c. commercial $(<$ commerce), amoureux ( $<$ amour $)$ [français]

Le second argument de Véronique concerne la position attributive. Un adjectif épithète post-nominal pourrait être analysé comme étant à l'intérieur d'une proposition relative (Véronique 2000), ou relative 
réduite. L'exemple (11a) pourrait être analysé de deux manières. Les parenthèses et les étiquettes AP et $\mathrm{CP}$ représentent une formalisation des propositions de Véronique.
a. Jenny oule
enn asistan
malin.
J. vouloir
un assistant
intelligent
'Jenny veut un(e) assistant(e) intelligent(e).'
b. Jenny oule enn asistan [AP malin].
c. Jenny oule enn asistan [CP [C ø] [AP malin]].

L'adjectif malin se trouve soit dans la proposition principale comme en (11b), soit dans une proposition relative ((11c)) qui ne contient ni complémenteur, ni copule. Cette possibilité existe parce que le complémenteur (ou le mot subordonnant introduisant les relatives) peut être occupé par une catégorie vide.
oule asistan
J. vouloir assistant
$\{\mathrm{ki} / \varnothing\} \mathrm{koz}$
COMP parler
angle bien.
'Jenny veut un(e) assistant(e) qui parle bien l'anglais.'
Jenny oule enn asistan $\{\mathrm{ki} / \varnothing\}$
b. Jenny oule enn asistan $\{\mathrm{ki} / \varnothing\} \quad$ malin.
J. vouloir un assistant COMP intelligent
'Jenny veut un(e) assistant(e) intelligent(e).'

L'exemple (9a) montre que le complémenteur est facultatif, et l'exemple (9b) montre qu'on ne peut pas distinguer la modification adjectivale de la la modification par une relative. Cette ambiguïté structurale suggère que les adjectifs apparemment post-nominaux ne font pas partie de la liste des adjectifs du mauricien. Seuls les adjectifs pré-nominaux peuvent être qualifiés d'adjectifs de manière catégorique. Dans cette perspective, il n'y a pas de raison pour que le lexème malin ne soit pas classé parmi les verbes.

On peut démontrer que l'adjectif malin ne se trouve pas dans une relative réduite en faisant le test de coordination. Les exemples (10a-b) montrent que malin ne peut pas être coordonné avec une relative réduite. On ne s'attendrait pas à un tel résultat si malin était enchâssé dans une relative réduite.

On obtient la même agrammaticalité avec un SN défini (10c-d). Les SN relativisés définis continnent le déterminant la qui se place en position finale, après tous les modifieurs.

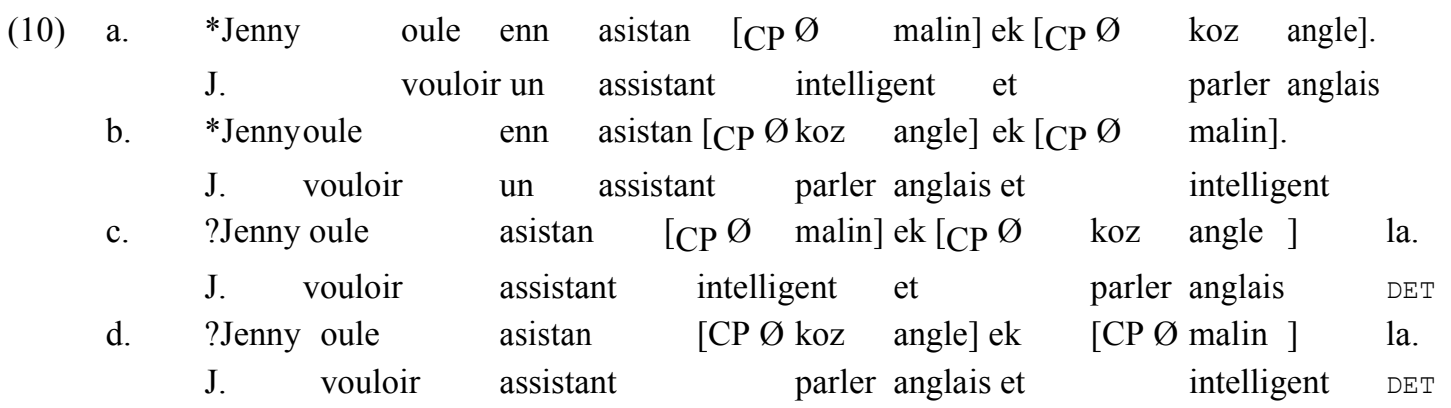

Ces données indiquent que la position épithète est accessible aux adjectifs en mauricien. 


\section{Caractéristiques des adjectifs en mauricien}

L'objectif dans cette section est de décrire les faits syntaxiques relatifs à la catégorie adjectivale. J'aborde en premier lieu des quantifieurs de degré, dont la plupart ne permettent pas de distinguer entre adjectifs et verbes. Le critère le plus important pour différencier les adjectifs des verbes est l'existence de verbescopules. Le second concerne certaines constructions causatives qui distinguent les adjectifs des verbes. Je terminerai par les combinaisons Aspect + Adjectif, qui montrent que les adjectifs et la marque du progressif sont incompatibles.

\subsection{Les quantifieurs de degré}

Certaines expressions de degré de l'anglais ne peuvent modifier que des adjectifs, notamment les marqueurs so 'si', too 'trop', as 'aussi', very 'très', enough 'assez'. Dans la typologie des expressions de degré de Doetjes (1997), ces expressions sont analysées comme étant les têtes d'une projection de Degré (Deg de DegPhrase). Ces expressions sont à distinguer des quantifieurs de degré pour lesquels il n'existe pas de contrainte sur le type de syntagme modifié. L'adverbe de degré trop, par exemple, se combine avec les adjectifs et les verbes. L'adverbe beaucoup se combine avec des formes au participe passé et avec les verbes. En comparant les expressions de degré en anglais, en français et en néerlandais, Doetjes (2008) conclut que des quantifieurs dédiés aux adjectifs sont rares.

En mauricien, il n'existe pas de quantifieur de degré réservé aux adjectifs. Les expressions suivantes peuvent se combiner à la fois avec les verbes et les adjectifs : bien 'très', tro 'trop', inpe 'un peu', osi 'aussi', ase 'assez', sitan 'tellement', telman 'tellement'. Les exemples en (11) illustrent ces deux possibilités pour le quantifieur bien.

(11) a. Rina bien for.

R. très fort

'Rina est très forte.'

b. Rina bien resanble so ser.

R. très ressembler POSS-3SG soeur

'Rina ressemble beaucoup à sa soeur.'

En y regardant de plus près, on remarque une différence dans l'ordre linéaire pour le quantifieur inpe. Alors qu'il peut occuper la position post-verbale ou pré-verbale, il est contraint à la position postadjectivale lorsqu'il modifie un adjectif. Même si les adverbes du mauricien ne sont pas spécialisés pour les adjectifs, la différence d'ordre linéaire constitue une différence entre la prédication verbale et la prédication adjectivale. 
(12) a. Sa dipin la inpe dir (*inpe). DEM pain DET un.peudur un.peu

'Ce pain est un peu dur.'

b. Rina inpe resanble so ser (inpe).

R. un.peuressembler POSS-3SG soeur un.peu

'Rina ressemble un peu à sa soeur.'

Cette différence peut s'expliquer par une différence d'interprétation. En effet, inpe est à la fois un quantifieur de degré et une expression de modalité épistémique. J'approfondirai cette question dans un futur travail.

Il est aussi important de noter que les quantifieurs de mesure ne peuvent se combiner avec des adjectifs. Le quantifieur enn tigit/ enn-tigin 'un petit peu' dans l'exemple (13a) quantifie sur le degré de ressemblance entre Rina et sa soeur, mais il ne peut se combiner avec l'adjectif kourt (13b).

$$
\begin{aligned}
& \text { a. Rina resanble so } \quad \text { ser enn-tigit. } \\
& \text { R. ressembler POSS-3SG } \\
& \text { 'Rina ressemble un petit peu à sa soeur.' } \\
& \text { b. Jenny enn-tigin kourt. } \\
& \text { J. un.petit.peu court } \\
& \text { 'Jenny est un peu petite.' }
\end{aligned}
$$

\subsection{Les verbes-copules}

Dans cette section, il est question de la distribution de vinn 'devenir' et de res 'rester/continuer'. J'ai mentionné dans la section 3 le fait que le mauricien n'emploie pas de copule pour la prédication adjectivale. Cependant, le mauricien possède (au moins) un verbe-copule, vinn 'devenir' qui accepte des compléments adjectivaux et qui ne se combine pas avec des compléments verbaux.

$\begin{array}{llll}\text { a. Anil pou vinn } & \text { long. } \\ & \text { Anil MOD devenir } & \text { grand } \\ & \text { 'Anil deviendra grand.' } & \\ \text { b. } & \text { *Anil pou vinn } & \text { reflesi } \\ & \text { Anil MOD devenir } & \text { réfléchir } \\ \text { c. Anil pou vinn enn } & \text { dokter . } \\ & \text { Anil MOD devenir } & \text { un médecin } \\ & \text { 'Anil deviendra médecin.' }\end{array}$

Le verbe-copule vinn accepte aussi des compléments nominaux si ceux-ci sont déterminés. Il s'agit ici d'un parallélisme entre la catégorie adjectivale et la catégorie nominale. Le fait de sélectionner des adjectifs est une propriété sémantique de vinn qui est la traduction de become. Le verbe vinn est l'expression du concept BECOME et ce type de verbes sont des fonctions qui s'appliquent à des états. Selon la terminologie de Jackendoff (1990), la Lexical Conceptual Structure de vinn est 'BECOME [STATE]' où l'état est dénoté par un adjectif. Ce critère est cependant limité aux adjectifs qui dénotent des états temporaires, et exclut les adjectifs dénotant des propriétés permanentes ou intrinsèques. 
Il est aussi intéressant de regarder a distribution du second verbe-copule res. C'est un verbe étymologiquement dérivé du verbe français rester. Il est à la fois un verbe copulatif qui est employé avec les locatifs et les adjectifs (15a-b), et un auxiliaire ayant le sens de 'continuer à'. Il se combine directement avec les adjectifs, et lorsqu'il se combine avec les verbes, il est accompagné de l'intensif mem et il participe parfois d'une construction sérielle avec ale 'aller'. Cela pourrait être rajouté à la liste des différences entre verbes et adjectifs.

a. Mala res lakaz.
M. rester maison
'Mala reste à la maison.'
b. Rina res meg.
$\begin{aligned} & \text { R. rester mince } \\ & \text { 'Rina reste mince.' }\end{aligned}$
c. Jenny res ekrir (ale) mem.

J. AU AU écrire aller INTENS

'Jenny continue à écrire (sans s'arrêter).' [res V]

\subsection{Les structures causatives}

Le mauricien distingue les structures causatives dont le complément est un verbe et les structures causatives dont le complément est un adjectif. La structure fer vinn 'faire devenir' se construit avec un adjectif et exclut les verbes. Quant au verbe fer 'faire', il se construit avec un verbe et exclut les adjectifs. Ce contraste est illustré en (16) et (17).
a. Traka pe fer mo
seve
vinn
blan.
souci PROG faire POSS-1SG cheveu
devenir blanc

'Les soucis rendent mes cheveux blancs.' Litt. 'Les soucis font mes cheveux devenir blancs.'
b. *Trakape fer mo seve blan.
souci PROG faire POSS-1SG cheveu blanc
a. Sa lakrem la fer mo
seve pouse.
DEM crème DET faire POSS-1SG
cheveupousser
'Cette crème fait pousser mes cheveux.'
b. $*$ Sa lakrem la fer mo
$\mathrm{DEM}$ crème DET faire POSS-1SG

$\begin{array}{lll}\text { seve } & \text { vinn } & \text { pouse. } \\ \text { cheveu } & \text { devenir } & \text { pousser }\end{array}$

\subsection{Les constructions 'Aspect + Adjectif'}

Les marques d'aspect du mauricien sont le progressif pe et le perfectif inn. Certains auteurs soutiennent que la compatibilité avec le progressif indique que les adjectifs font partie de la classe des verbes (cf. Alleyne 1996: 64 et Véronique 1983: 208). Je soutiens l'hypothèse inverse : une des caractéristiques des adjectifs en mauricien est l'incompatibilité avec le progressif. Cette généralisation recouvre la grande majorité des adjectifs. Véronique (2000) remarque que la possibilité de se combiner avec le progressif est limitée à quelques items lexicaux et il conclut qu'il serait erroné d'en faire une propriété générale des 
adjectifs. Les lexèmes qu'il cite dénotent tous deux des états mentaux, mais il n'y en a qu'un seul qui puisse se combiner avec le progressif.

(18) a. * $\mathrm{Li}$ pe kouyon.

3SG PROG stupide

b. $\mathrm{Li}$ pe toke.

3SG PROG fou

'Il/Elle devient fou.'

Les adjectifs/verbes qui sont compatibles avec le progressif se limitent à la liste suivante:

(19) mir 'mûr/mûrir', fay 'en mauvaise forme/affaibli', fre 'froid/refroidir', fou 'fou' et son synonyme toke 'fou'.

Un tel phénomène peut s'analyser de deux manières. Selon Baker (2003 : chapitre 2), les marqueurs TMA de certaines langues seraient à la fois compatibles avec les verbes et les adjectifs. Dans les langues où c'est le cas, ce sont les propriétés sélectionnelles de ces morphèmes qui leur donnent une plus grande flexibilité. Le marqueur perfectif inn, qui possède la propriété de se combiner avec des adjectifs et des verbes, fait partien selon moi, de ces marqueurs non-sélectifs.

Le fait que le progressif pe ne se combine qu'avec des lexèmes spécifiques ne constitue pas un argument suffisamment convaincant pour classer tous les adjectifs dans la classe des verbes. A la différence de Baker qui avance l'idée de sous-spécification catégorielle pour les marqueurs TMA, j'estime que l'hypothèse la plus simple pour le progressif est de traiter les lexèmes en (22) comme des expressions de catégorie indéterminée. Elles ont la possibilité d'être employées soit comme des adjectifs, soit comme des verbes. Leur catégorisation dépend du contexte syntaxique (ce qui est reflétée dans la double glose).

Ainsi, le terme mir 'mûrir/mûr' change d'interprétation et de catégorie selon la construction syntaxique dans lequel il se trouve.

$\begin{array}{lll}\text { a. } & \text { Mang la pe mir. } \\ \text { mangue } & \text { DET PROG mûr } \\ \text { 'La mangue est en train de mûrir.' } \\ \text { b. } & \begin{array}{l}\text { Mang la inn mir. } \\ \text { mang DET PERF mûr }\end{array} \\ & \text { 'La mangue est (maintenant) mûre.' } \\ \text { c. } & \text { Mang la mir. } \\ & \text { mang } \text { DET mûr } \\ & \text { 'La mangue est mûre.' }\end{array}$

La phrase en (22a) décrit un procès où la mangue progresse sur une échelle de maturation au fil du temps. Levin et Rappaport Hovav (2005: 95) décrivent ce procès en ces termes : "Progress along the scale is correlated with progress through the event, and the end of the scale provides the point of delimitation of the event. » (d'après l'analyse de Tenny 1994 : 17-18). En conclusion, la généralisation que les adjectifs prennent le progressif est erronnée. La grande majorité des adjectifs du mauricien sont incompatibles avec le progressif, ce qui en fait un critère définitoire de cette classe. Il est question dans ce qui suit des constructions avec le perfectif. 
Le perfectif se retrouve à la fois les verbes et les adjectifs, mais il ne produit pas la même interprétation. Dans les constructions perfectif + adjectif, l'interprétion se fait selon le point-de-vue résultatif. Le syntagme adjectival décrit un état qui est le résultat d'un procès comme en (21a). La construction perfectif $+V$ décrit un événement de son point de vue final.

$$
\begin{array}{lllll}
\text { a. Zanfan } & \text { la } & \text { inn } & \text { gran. } & \\
\text { enfant } & \text { DET } & \text { PERF } & \text { grand } \\
& \text { 'L'enfant est maintenant (plus) grand.' } & \text { [point-de-vue résultatif] } \\
\text { b. } & \begin{array}{l}
\text { Zanfan } \\
\text { enfant la }
\end{array} & \text { inn } & \text { grandi. } & \\
& \text { 'L'enfant a grandi.' } & \text { PERF } & \text { grandir } & \\
\end{array}
$$

La construction 'inn + adjectif' décrit un état situé au moment de l'énonciation. Dans la construction inn + grandi, l'événement est situé dans le passé et il est appréhendé du point de vue perfectif. On peut vérifier la localisation temporelle en posant la question 'Quand ?' Cette possibilité existe dans les cas de prédication verbale, mais elle est exclue avec les adjectifs.

$\begin{array}{llllllll}\text { a. } & \text { Zanfan } & \text { la } & \text { inn } & \text { gran. *Kan } & \text { li' } & \text { nn } & \text { gran ? } \\ & \text { enfant } & \text { DET } & \text { PERF } & \text { grand quand } & \text { 3SG } & \text { PERF } & \text { grand } \\ \text { b. } & \text { Zanfan } & \text { la } & \text { inn } & \text { grandi. } & \text { Kan li' } & \text { nn } & \text { grandi ? } \\ & \text { enfant } & \text { DET } & \text { PERF } & \text { grandir } & \text { quand 3SG } & \text { PERF } & \text { grand } \\ & \text { 'L'enfant a grandi. Quand est-ce qu'il a grandi ?' } & & \end{array}$

Les critères que j'ai présentés dans cette section permettent d'isoler les adjectifs qui décrivent une propriété d'un individu. Pour qu'un item lexical puisse être le complément de vinn 'devenir', il faut qu'il décrive une propriété. C'est le cas à la fois des adjectifs et des noms qui dénotent des propriétés.

(23) Zan inn vinn dokter.

J. PERF devenir médecin

'Jean est devenu médecin.'

Ce critère ne peut s'appliquer à des états temporaires (comme malad 'malade') ou des états psychologiques, qui sont des items compatibles avec le progressif. Cette étude doit donc être complétée par une comparaison entre ces derniers et les adjectifs qui dénotent une propriété.

\section{Conclusion}

Cet article présente des critères distributionnels qui permettent de distinguer une classe adjectivale des verbes en mauricien. Il présente en ce faisant des arguments contre l'hypothèse que les adjectifs sont une sous-classe de verbes. Les critères que je retiens pour identifier les adjectifs sont la compatibilité avec le verbe-copule vinn et l'incompatibilité avec le progressif. D'autres différences concernent les constructions syntaxiques avec le verbe-auxiliaire res et les structures causatives. Concernant les quantifieurs de degré et la prédication seconde, les faits sont moins manquants et méritent d'être approfondis par des recherches futures. 


\section{Références bibliographiques}

Alleyne, M. C. (1996). Syntaxe historique créole. Paris: Editions Karthala.

Baker, M. C. (2003). Lexical categories: Verbs, nouns, and adjectives. Cambridge, UK: Cambridge University Press.

Cabredo-Hofherr, P. (2010). Adjectives: an introduction. In Cabredo-Hofherr \& O. Matushansky (sld.) Adjectives: formal analyses in syntax and semantics. Amsterdam/Philadelphia: John Benjamins. 1-26

Dixon, R. M.W. (1977). Where have all the adjectives gone? Studies in Language, 1, 19-80.

Dixon, R. M.W. (2004). Adjective classes in typological perspective. In Dixon, R. M.W. 2004 \& A. Aikhenvald (sld.) Adjective classes: a cross-linguistic typological study. Oxford : Oxford University Press. 1-49.

Doetjes, J.. (1997). Quantifiers and selection. La Haye : HIL.

Doetjes, J. (2008). Adjectives and degree modification. In McNally L. \& C. Kennedy (sld.) Adjectives and adverbs: syntax, semantics, and discourse. Oxford : Oxford University Press. 123-155.

Haspelmath, M. (2007). Pre-established categories don’t exist. Consequences for language description and typology. Linguistic Typology, 11, 119-132.

Jackendoff, R. (1990). Semantic structures. Cambridge, Mass. : MIT Press.

Holm, J. (2000). An introduction to pidgins and creoles. Cambridge: Cambridge University Press.

Kennedy, C. (2012). Adjectives. In Russell G. \& D. Graff Fara (sld.) Routledge companion to philosophy of language. 328-341.

Levin, B. \&. M. Rappaport Hovav. (2005). Argument realization. [Research surveys in Linguistics] Cambridge: Cambridge University Press.

Maurer, P \& APiCS Consortium. (2013). Gender agreement of adnominal adjectives. In: Michaelis, S. M., P. Maurer, M. Haspelmath, M. Huber (sld.) Atlas of Pidgin and Creole Language Structures Online. Leipzig: Max Planck Institute for Evolutionary Anthropology. (Available online at http://apics-online.info/parameters/40, Accessed on 2014-02-25.)

Véronique, D. (1983). Existe-t-il une classe adjectivale en mauricien ? CLAIX. Travaux 1. Les parties du discours. 201-222.

Véronique, D. (2000). Notes sur les « adjectifs » dans quelques créoles français. Langages, 138, 61-69. 